\title{
Optimal portfolio allocation under the probabilistic VaR constraint and incentives for financial innovation
}

\author{
Jón Daníelsson • Bjørn N. Jorgensen • \\ Casper G. de Vries • Xiaoguang Yang
}

Received: 10 April 2006 / Accepted: 6 July 2007 / Published online: 21 August 2007

(C) Springer-Verlag 2007

\begin{abstract}
We characterize the investor's optimal portfolio allocation subject to a budget constraint and a probabilistic VaR constraint in complete markets environments with a finite number of states. The set of feasible portfolios might no longer be connected or convex, while the number of local optima increases exponentially with the number of states, implying computational complexity. The optimal constrained portfolio allocation may therefore not be monotonic in the state-price density. We propose a type of financial innovation, which splits states of nature, that is shown to weakly enhance welfare, restore monotonicity of the optimal portfolio allocation in the state-price density, and reduce computational complexity.
\end{abstract}

We are grateful to Ken Kavajecz and seminar participants at Harvard Business School, London School of Economics, Maastrict University, ZEI Bonn, and Danske Bank Symposium on Asset Allocation and Value-at-Risk: Where Theory Meets Practice for comments on an earlier version of this paper. We also benefitted from the suggestions of two anonymous referees. Our papers can be downloaded from www.RiskResearch.org.

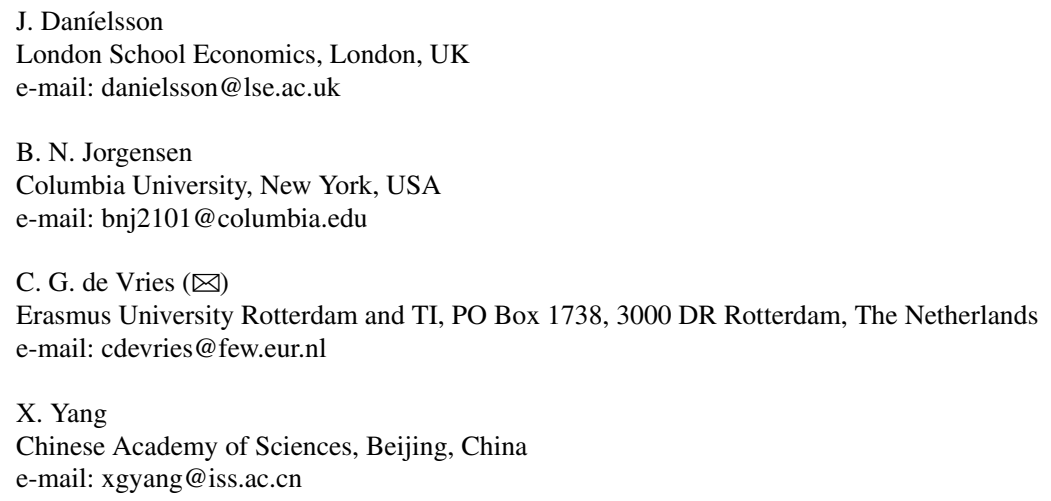


Keywords Portfolio optimization - Value-at-risk - Computational complexity • State-price density

\section{JEL Classification G11}

\section{Introduction}

Economists have formally addressed the problem of portfolio optimization subject to a budget constraint starting with Markowitz (1952) and Roy (1952). Roy's criterion delimits the probability of highly adverse outcomes and maximizes expected return, while Markowitz' solution trades off the mean return against the variance as a risk indicator. Though less popular than the mean variance tradeoff, the concern for the downside risk aspect remained, as evidenced by Markowitz's (1959) contribution using the semi-variance, Arzac and Bawa's (1977) extension of Roy's safety first criterion and Leland's (1980) portfolio insurance. The downside risk measures received renewed attention with the official adoption of the Value-at-Risk (VaR) measure in the Basel capital accords and the attention for downside risk concerns in behavioral finance. Consequently VaR has emerged as an important side constraint on investment behavior.

We formally analyze the portfolio optimization problem within the expected utility function framework, subject to a budget constraint and a VaR constraint i.e., a probabilistic constraint. The analysis is set in an economy with a finite number of states and a complete set of elementary securities. The special case of our problem is the portfolio insurance problem, which has been analyzed before. The incorporation of a VaR constraint both complicates the analysis and has interesting implications. We show that the optimal constrained portfolio allocation may not be monotonic in the state-price density and that this provides a new rationale for financial innovation.

The prior literature on financial innovations recognizes three distinct categories of innovation. Initial models document how financial innovation is used to complete markets, see Ross (1976). The second category is strictly within the incomplete market paradigm, i.e., even after the introduction of new securities the market remains incomplete. In this case, some investors have an incentive to issue new securities, but this may not improve efficiency (See e.g., Allen and Gale 1988, 1991). A third category discusses financial innovation in a complete market setting where the marketable securities, which are bundles of (non-traded) elementary securities, achieve complete spanning. In this case, regulation prohibits unlimited trading in available securities. Miller $(1986,1992)$ argues that financial innovation is often driven by regulation, e.g. short sale restrictions. Such trading friction gives an incentive for financial innovation via unbundling of states, in order to achieve the Pareto optimal free market outcome through trading the elementary securities. A representative example of this approach is Chen (1995). Grinblatt and Longstaff (2000) find that stripping and trading separately the principal and coupons of treasury bond helps complete markets and overcome frictions, which explains why rebundling is observed simultaneously with unbundling.

In this paper we introduce a fourth category of financial innovations in complete markets, where all elementary securities are freely traded. We demonstrate that an incentive for financial innovation may remain whereby elementary securities are split 
up and new states are artificially created through a randomizing devise. We show that the demand for this type of innovation is triggered by the imposition of nondeterministic downside risk constraints, such as VaR. In this case, the splitting of states can help in reducing the negative effects of risk constraints on expected utility. An example of this type of securities, discussed below, are the lottery bonds issued by several European governments. Lottery bonds are bonds where a randomizing device is used to determine which bonds are called in early or which bonds pay a coupon. We show that such type of securities may simplify the investor's portfolio allocation problem.

We formally demonstrate that the portfolio problem with a probabilistic downside risk constraint is computationally intractable when the state space is finite, but large, i.e., is NP-complete. ${ }^{1}$ We proceed to show that investors can simplify their portfolio allocation problem and increase welfare through financial innovation that entails introducing additional redundant securities which are lotteries over existing elementary Arrow-Debreu securities. By splitting existing states, we show that not only is utility increased, but also that an otherwise complex problem can be rendered solvable, in the sense that it takes only a polynomial number of steps so that the problem is in class $P$.

While we consider the optimal portfolio allocation for any value of VaR risk level $\delta \in[0,1]$, the preceding literature has focused on the two extreme values, $\delta=1$ and $\delta=0$. First, consider traditional portfolio allocation where $\delta=1$, such that the risk constraint is never binding. Solving such problems does not introduce any significant difficulty as standard methodology readily applies (see e.g. Kuhn and Tucker 1951; Uzawa 1958) and a global optimum is ensured for any strictly concave utility function. It follows that this portfolio problem is readily solved in polynomial time.

Second, the case of $\delta=0$ corresponds to portfolio insurance with a deterministic floor constraint such that losses cannot fall below the exogenously determined level, $K$. Grossman and Vila (1989) demonstrate that the solution to this problem is straightforward, where in effect the investor buys a put option with exercise price $K$. We demonstrate below that the portfolio insurance problem is computationally simple, i.e., solvable in polynomial time. An early example of a non-deterministic constraint is the safety-first portfolio selection program as analyzed in the Arzac and Bawa (1977) lexicographic interpretation. However, safety-first may lead to strained portfolio choices: A portfolio manager may allocate just enough to the safe asset to meet the downside risk constraint, while the remainder of funds is invested in the option with the highest available strike price (see Dert and Oldenkamp 1997; Vorst 2000).

\footnotetext{
1 NP-complete (non-deterministic polynomial complete) is a term used in complexity theory to identify a particular class of problems. For an NP-complete problem, it is generally believed that the relationship between the number of input parameters to the problem and the problem complexity is exponential, that is, the computational time for solving the problem increases exponentially with the size of the problem. For example, the travelling salesman problem and the partition problem are NP-complete. For a formal definition of NP-completeness, see chapt. 36 in Cormen et al. (1999) or Papdimitriou and Steiglitz (1982, chapt.15). If the solution only requires a polynomial number of steps, the problem is in the class $\mathrm{P}$.
} 
The general case considered in this paper, allows $\delta$ to take any value between zero and one. This is the VaR problem where the investor does not want wealth to fall below level $K$ with more than a given probability $\delta$. We demonstrate that this problem is computationally complex unless the number of states of nature is sufficiently small or the VaR constraint is non-binding. The reason for the complexity is that the set of feasible portfolios is no longer convex, in fact, the budget set has disjoint components, each containing a local optimum. In an economy with few states it is straightforward to consider all components to find the solution. However, in general, the number of local maxima can increase exponentially in the number of states, i.e., the number of local maxima may approach $n$ !.

We show how such a non-deterministic downside risk constraint may also change the qualitative aspect of the solution in an important way. It is well known that the optimal portfolio is monotonic in the state price density when $\delta=0$ or $\delta=1$. Basak and Shapiro (2001) conclude that VaR constrained optimal portfolio allocations are monotonic with respect to the state price density in a Brownian motion setting with continuous rebalancing. In contrast, we show that the optimal portfolio allocation can be non-monotonic in the state price density when the state space is discrete. Furthermore, for the discrete state space problem we derive a sufficient condition such that the optimal portfolio allocation of the discrete problem is monotonic in the state price density. Finally, we show that investors have an incentive by means of financial innovation to create seemingly redundant securities to meet this sufficient condition. These new securities render the initial complex problem solvable, since the monotonicity in the state price density guarantees polynomial time solutions.

In the special case where the probabilities and prices can be weakly reverse ordered, the VaR constrained problem is simple. Under this reverse ordering condition we can write the risk constraint as a restriction on price only. In effect under this condition, the VaR constrained problem is reduced to a generalized portfolio insurance problem. Consider the special case of uniform probabilities. Since probabilities do not play a role for any $\delta$, the intuition is immediate. Under the reverse ordering condition, we show that the risk constrained problem enables polynomial time solutions by employing techniques similar to Grossman and Vila (1989). When state splitting is feasible, states can be split such that our reverse ordering condition is satisfied. Relative to the augmented state space, the problem has a polynomial time solution. We also demonstrate the positive impact of state splitting in an example where the VaR constraint is met optimally by investing in one of the two new states. Subsequent to state splitting, the optimal portfolio is monotonic in the state price density.

The structure of the paper is as follows. In Sect. 2 we demonstrate that the risk constrained problem is computationally complex. Section 3 first analyzes portfolio insurance and then introduces the reverse ordering condition. We show that under the reverse ordering condition, the solution to the VaR constrained problem is simple. In Sect. 4 we discuss the drive for financial innovation to bring about the reverse ordering condition. Section 5 concludes the paper. Two examples are used throughout the text to illustrate our ideas. The examples offer a roadmap for the theoretical developments. 


\section{Utility maximization and risk constraints}

We consider a single period complete market spanned by $n$ Arrow-Debreu securities. The investor with initial wealth, $W$, can purchase for price $p_{i}$ contingent claims on each state, $i=1, \ldots, n$, which pay out $\$ 1$ in state $i$ at the investment horizon and zero otherwise. State $i$ occurs with probability $\pi_{i}$. We consider an investor whose preferences are characterized by a strictly increasing, concave expected utility function in wealth. The investor's problem is choosing portfolio $\left\{x_{i}\right\}_{i=1}^{n}$ where $x_{i}$ denotes the number of security $i$ purchased.

\subsection{Risk restrictions}

Public policy often restricts the risk that can be assumed by a strict subset of all investors. For example, national supervisory authorities impose VaR constraints on commercial banks in order to contain market risk. Other financial intermediaries like pension funds work with self-imposed constraints like portfolio insurance where the investors' net worth is never allowed to fall below a certain predetermined level $K$. The formal definition of the VaR constraint is as follows.

Definition 1 (Value-at-Risk) VaR is the zero'th lower partial moment. For any discrete distribution, the VaR constraint can be written as

$$
\sum_{i=1}^{n} 1_{\left\{x_{i} \leq K\right\}} \pi_{i} \leq \delta
$$

where the indicator function in the VaR constraint is with respect to state $i .^{2}$

To ensure the existence of a finite solution to the investor's portfolio choice problem, $\left|x_{i}\right|<\infty, \forall i$, two different approaches have been suggested. First, one can rule out unlimited borrowing. For presentational simplicity, we simply rule out all short sales in Arrow-Debreu securities. However, all results go through with an arbitrary, finite lower bound on short selling. Moreover note that this does not constrain investors from short selling non-elementary securities. Also note that this does not affect the feasibility

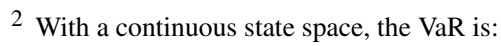

$$
\int_{-\infty}^{K} f(x) d x=\int_{-\infty}^{\infty} 1_{\{x \leq K\}} f(x) d x=\delta
$$

to allow for unordered integrands. 
of the VaR problem. ${ }^{3}$ Alternatively, we could impose mild regularity conditions on the investor's utility function, such as the Inada conditions or the self-concordance condition, see Ingersoll (1987, p. 189), and den Hertog (1995) respectively. Since all examples below adhere to these regularity conditions, the short selling restriction is not driving our results. Our investor's problem can be stated as:

\section{Problem 1}

$$
\begin{aligned}
& \max _{\left\{x_{i}\right\}_{i=1}^{n}} \sum_{i=1}^{n} \pi_{i} u\left(x_{i}\right) \quad \text { s.t. } \quad \sum_{i=1}^{n} p_{i} x_{i} \leq W \\
& \sum_{i=1}^{n} 1_{\left\{x_{i} \leq K\right\}} \pi_{i} \leq \delta \\
& x_{1}, x_{2}, \ldots, x_{n} \geq 0
\end{aligned}
$$

This implies that the probability that the value of the investor's portfolio falls below $K$ at the investment horizon cannot exceed $\delta$. While we consider any value of $\delta \in[0,1]$, the preceding literature has focused on the two extreme values, $\delta=1$ and $\delta=0$.

\subsection{The standard portfolio choice problem}

Consider first the standard portfolio choice problem with $\delta=1$, which we refer to as the unconstrained case because the VaR constraint is never binding. Let $\lambda$ denote the Lagrange multiplier associated with the budget constraint which is binding. An interior solution, if it exists, is characterized by the budget constraint and

$$
x_{i}=\left(u^{\prime}\right)^{-1}\left(\lambda p_{i} / \pi_{i}\right), \quad \forall i
$$

Without loss of generality, the states can then be ordered such that

$$
\lambda p_{i} / \pi_{i} \geq \lambda p_{i+1} / \pi_{i+1}, \quad \forall i
$$

3 Ruling out infinite shortsales is equivalent to preventing infinite borrowing. Impose restriction, $B$, on borrowing then the investor's problem can be stated as:

$$
\min _{\left\{x_{i}\right\}_{i=1}^{n}} \sum_{i=1}^{n} 1_{\left\{x_{i} \leq K\right\}} \pi_{i} \quad \text { s. t. } \begin{aligned}
\sum_{i=1}^{n} p_{i} x_{i} & =W \\
x_{1}, x_{2}, \ldots, x_{n} & \geq-B
\end{aligned}
$$

Let $y_{i}=x_{i}+B$, then this problem is equivalent to

$$
\min _{\left\{y_{i}\right\}_{i=1}^{n}} \sum_{i=1}^{n} 1_{\left\{y_{i} \leq B+K\right\} \pi_{i}} \text { s. t. } \begin{aligned}
\sum_{i=1}^{n} p_{i} y_{i} & =W+B \sum_{i=1}^{n} p_{i} \\
y_{1}, y_{2}, \ldots, y_{n} & \geq 0
\end{aligned}
$$

which for the purpose of the arguments in this paper is equivalent to ruling out short sales. 
or, equivalently, such that

$$
x_{i} \leq x_{i+1}, \quad \forall i
$$

Given the assumptions above, the first-order conditions guarantee that (2) is a globally optimal solution when the utility function is strictly concave and the domain is a bounded convex set.

\subsection{The portfolio insurance problem}

Secondly, consider the portfolio choice problem with $\delta=0$. Grossman and Vila (1989) demonstrate that this portfolio insurance problem has the intuitively appealing feature that the investor buys a put with exercise price $K$. Further details of the solution are discussed in Sect. 3. The more recent VaR constraint, discussed in the next subsection, is an extended portfolio insurance problem where the investor's net worth cannot fall below $K$ with more than $\delta$ probability. To formalize our analysis we introduce terminology from operations research.

\subsection{Solution complexity}

In order to characterize the computational complexity, we relate the current Problem 1 to the partition problem since the partition problem is known to be NP-complete, see Papdimitriou and Steiglitz (1982, chapt. 15). If a problem can be reduced to another problem which is known to be a member of the NP-complete class, it follows that this problem is also a member of this class. Furthermore, NP-completeness also implies that checking the correctness of an answer must be possible in a polynomial number of steps.

Definition 2 (Partition problem) Let $\left\{a_{1}, a_{2}, \ldots, a_{n} ; b\right\}$ be $n+1$ positive numbers such that $\sum_{i=1}^{n} a_{i}=2 b$. Does a subset $S \subset\{1,2, \ldots, n\}$ exist such that $\sum_{i \in S} a_{i}=b$ ?

Theorem 1 The feasibility of Problem 1 is NP-complete.

Proof It is trivial to see that the feasibility of Problem 1 is NP, i.e., checking answers takes only a polynomial number of manipulations. To establish NP-completeness, we only need show that the problem can be reduced to the partition problem. From a given instance of the partition problem, let us construct an instance of Problem 1. Let $\pi_{i}=\frac{a_{i}}{2 b}, p_{i}=a_{i}, 1 \leq i \leq n$. Let $K=b, W=b^{2}$ and $\delta=\frac{1}{2}$. We claim that the partition problem has a feasible solution if and only if Problem 1 has a feasible solution. In fact, if the partition problem has a feasible solution $S$, that is $\sum_{i \in S} a_{i}=b$, we set $x_{i}=b$ if $i \in S$ and $x_{i}=0$ otherwise. Then we have $\sum_{i=1}^{n} p_{i} x_{i}=b^{2}$, $\sum_{x_{i} \geq K} \pi_{i}=\frac{1}{2}$. Thus $x$ is a feasible solution of Problem 1. Conversely, assume $x$ is a feasible solution of Problem 1. We have $\sum_{i=1}^{n} p_{i} x_{i}=b^{2}$ and $\sum_{x_{i} \geq K} \pi_{i} \geq \frac{1}{2}$. Denote 
$S=\left\{1 \leq i \leq n \mid x_{i} \geq K\right\}$. We have

$$
\begin{aligned}
& b^{2}=\sum_{i=1}^{n} p_{i} x_{i} \geq \sum_{i \in S} p_{i} b \\
& \sum_{i \in S} \pi_{i}=\sum_{x_{i} \geq K} \pi_{i} \geq \frac{1}{2}
\end{aligned}
$$

Using $p_{i}=a_{i}$ and $\pi_{i}=a_{i} / 2 b$ we get

$$
\begin{aligned}
& b \geq \sum_{i \in S} a_{i}, \\
& \sum_{i \in S} a_{i} \geq b
\end{aligned}
$$

Hence we obtain $\sum_{i \in S} a_{i}=b$. This means that $S$ is a feasible solution of the partition problem.

It follows that finding the solution to the VaR constrained portfolio choice Problem 1 is computationally complex since NP-complete problems are generally believed to not be solvable in polynomial time. Hence, the optimal portfolio can be computed when $n$ is sufficiently small or the problem is otherwise simple (see below). In general the number of local maxima may aproach $n$ !, i.e., the number of potential local maxima increases exponentially in the number of states.

Note that the proof of Theorem 1 allows for a risk neutral investor with limited short sales. Thus, even when the lexicographic interpretation of the safety-first criterion, as by Arzac and Bawa (1977), is feasible, it is also burdened by the computational complexity.

\subsection{Characterizing the optimal portfolio}

The cause for the computational complexity that may arise due to the VaR side constraint is most easily explained by means of an example. This example will also be used later in the paper to demonstrate the incentives for financial innovation.

Example 1 Consider a setting with a VaR constraint and logarithmic utility, and two states of nature.

$$
\begin{aligned}
\max _{x_{1}, x_{2}} \pi_{1} \log \left(x_{1}\right)+\pi_{2} \log \left(x_{2}\right) & \\
\text { s.t. } & p_{1} x_{1}+p_{2} x_{2} \leq W \\
& \leq W \\
1_{\left\{x_{1} \leq K\right\}} \pi_{1}+1_{\left\{x_{2} \leq K\right\}} \pi_{2} & \leq \delta
\end{aligned}
$$

Among the three possible orderings of probabilities and constraints, we consider the case where $1>\delta>\max \left(\pi_{1}, \pi_{2}\right)>0$, see Fig. 1 . In the figure $L$ represents the unconstrained portfolio allocation that would arise in the absence of a VaR constraint, which of course it violates. Due to the VaR constraint, the relevant budget constraint 


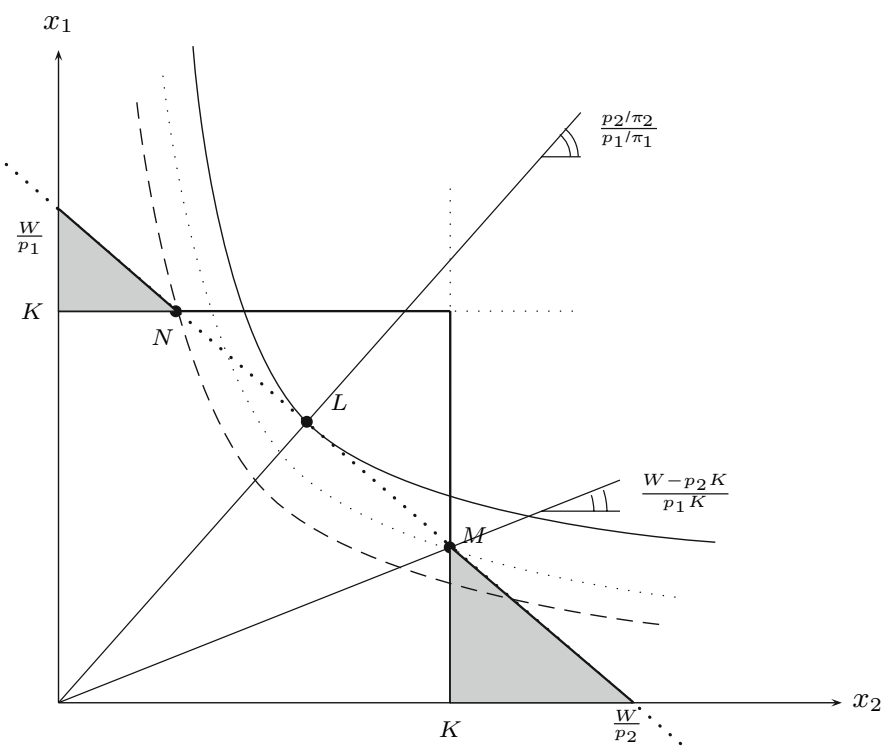

Fig. 1 Concave utility and the VaR constraint. Either $x_{1}$ or $x_{2}$ must satisfy the risk level $K$. The budget constraint is between points $W / p_{1}$ and $W / p_{2}$. Due to the shortsale restriction, negative values are ruled out, and the line segment between $N$ and $M$ is not feasible due to the VaR constraint. Hence the budget constraint is the two disjoint pieces $W / p_{1}-N$ and $M-W / p_{2}$. Note $x_{2}$ is drawn along the horizontal axis

is the two disjoint line segments $\left(0, W / p_{1}\right)-N$ and $M-\left(W / p_{2}, 0\right)$. These segments are individually convex, but not jointly, hence the Kuhn-Tucker conditions may only give a local solution since there are two local optima.

We can write the VaR constraint as a product, since either $x_{1} \geq K$ or $x_{2} \geq K$ :

$$
\begin{aligned}
\mathcal{L}= & \pi_{1} \log \left(x_{1}\right)+\pi_{2} \log \left(x_{2}\right) \\
& +\lambda\left\{W-p_{1} x_{1}-p_{2} x_{2}\right\} \\
& +\psi\left\{-\left(x_{1}-K\right)\left(x_{2}-K\right)\right\}
\end{aligned}
$$

The necessary Kuhn-Tucker conditions are

$$
\begin{aligned}
\pi_{1} \frac{1}{x_{1}}-\lambda p_{1}-\psi\left(x_{2}-K\right) & =0 \\
\pi_{2} \frac{1}{x_{2}}-\lambda p_{2}-\psi\left(x_{1}-K\right) & =0 \\
\lambda\left\{W-p_{1} x_{1}-p_{2} x_{2}\right\} & =0 \\
\psi\left\{-\left(x_{1}-K\right)\left(x_{2}-K\right)\right\} & =0 \\
\lambda \geq 0, \psi & \geq 0
\end{aligned}
$$

Consider a specific parameterization. Assume $\pi_{1}=2 / 3,2 / 3<\delta<1, p_{1}=1 / 3$, $p_{2}=1 / 4, W=1 / 4$, and $K=23 / 32$. In the unconstrained case, the optimal portfolio allocation would be $x_{1}=1 / 2$ and $x_{2}=1 / 3$, which is represented by the point $L$ in 
Fig. 1. To meet the VaR constraint, suppose we adjust the cheaper state, i.e., raise $x_{1}$ to $K$ (this appears to be cheaper because $x_{1}$ is closer to $K$ than $x_{2}$ and has a lower state price probability ratio, since $\left.p_{1} / \pi_{1}=1 / 2<p_{2} / \pi_{2}=3 / 4\right)$. This position is labelled as $N$ in Fig. 1. Alternatively, we could raise $x_{1}$ to $K$, the resulting portfolio allocation is labelled as $M$ in Fig. 1. The following Table summarizes the portfolio allocations and expected utilities:

\begin{tabular}{lccc}
\hline Maxima & $x_{1}$ & $x_{2}$ & $E U$ \\
\hline Unconstrained $(L)$ & $1 / 2$ & $1 / 3$ & -0.83 \\
VaR constrained $(N)$ & $23 / 32$ & $1 / 24$ & -1.28 \\
$\operatorname{VaR}$ constrained $(M)$ & $27 / 128$ & $23 / 32$ & -1.15 \\
\hline
\end{tabular}

The unconstrained solution $L$ favors $x_{1}$ over $x_{2}$ since $p_{1} / \pi_{1}<p_{2} / \pi_{2}$ (the usual monotonicity property). The optimal VaR constrained portfolio upsets the usual ordering. Note that in the optimal VaR constrained solution, the ratio $x_{1} / x_{2}$ is only a function of prices, $p_{1}$ and $p_{2}$, and the VaR constraint $K$. In contrast, both prices and probabilities determine this ratio in the unconstrained solution. Since probabilities do not determine the $x_{1} / x_{2}$ ratio in the VaR constrained case, the monotonicity of the solution in the state price density suggested by Eqs. (2) and (3) can be upset. For this reason all constrained local optima have to be evaluated to determine the global maximum. We have shown by means of this two state example the following result.

Proposition 1 With a discrete number of states the VaR constrained optimal portfolio solution may be non monotonic with respect to the state price density.

Below we write a unified Lagrangian function with probabilistic integer variables. However, since such integer variables are non differentiable, solutions based on first order conditions are not feasible. With many states this remains a cumbersome exercise. Thus, with just two states we cannot fully do justice to the qualitative properties of the VaR constrained solution. Therefore, we also povide a four-state example.

Example 2 Let the investor's utility function be $u(x)=-1 / x$, which implies a relative risk aversion parameter of 2 . Let there be four states. The following table lists the state probabilities and state prices of the four elementary securities.

\begin{tabular}{cllll}
\hline Statistic & \multicolumn{4}{c}{ States } \\
\hline$i$ & 1 & 2 & 3 & 4 \\
$\pi_{i}$ & $1 / 100$ & $1 / 2$ & $9 / 100$ & $40 / 100$ \\
$p_{i}$ & $1 / 100$ & $1 / 8$ & $1 / 100$ & $1 / 40$ \\
\hline
\end{tabular}

Suppose wealth $W=39 / 90$. In the absence of a VaR constraint, the investor chooses $\left(x_{1}, x_{2}, x_{3}, x_{4}\right)=(10 / 9,20 / 9,30 / 9,40 / 9)$, which yields expected utility $E U=\sum_{i=1}^{4} \pi_{i} u\left(x_{i}\right)=-0.3510$. Note that since the state price density is $\left(p_{1} / \pi_{1}, p_{2} / \pi_{2}, p_{3} / \pi_{3}, p_{4} / \pi_{4}\right)=(1,1 / 4,1 / 9,1 / 16)$, the solution $x_{1} \leq x_{2} \leq x_{3} \leq$ $x_{4}$ is in conformity with (3). Suppose the VaR regulation stipulates $\delta=0.4999$ and 
$K=2.5$. The unconstrained solution does not meet the downside risk constraint, since both $x_{1}$ and $x_{2}$ are below 2.5. Following the rule of thumb implied by the state price density ordering, one would raise the consumption in the state 2 , as $x_{2}$ is closest to the VaR level 2.5. In that case the investor chooses $\left(x_{1}, x_{2}, x_{3}, x_{4}\right) \approx$ $(0.86,2.50,2.59,3.45)$, with expected utility $E U=\sum_{i=1}^{4} \pi_{i} u\left(x_{i}\right) \approx-0.3622$. Note this portfolio allocation is feasible and preserves the ordering of the state price density. This portfolio is, however, not the optimal solution. The investor is better off by taking $x_{1}=2.5$. This permits $\left(x_{1}, x_{2}, x_{3}, x_{4}\right) \approx(2.50,2.14,3.21,4.28)$, with expected utility $E U=\sum_{i=1}^{4} \pi_{i} u\left(x_{i}\right) \approx-0.3576$. Although this is the optimal VaR restricted portfolio, the allocation is again not monotonic with respect to the state price density since $x_{1}>x_{2}<x_{3}<x_{4}$, of Proposition 1 .

\begin{tabular}{|c|c|c|c|c|c|}
\hline \multirow[t]{2}{*}{ Statistic } & \multicolumn{4}{|c|}{ States } & \multirow[t]{4}{*}{$E U$} \\
\hline & 1 & 2 & 3 & 4 & \\
\hline$\pi_{i}$ & $1 / 100$ & $1 / 2$ & $9 / 100$ & $40 / 100$ & \\
\hline$p_{i}$ & $1 / 100$ & $1 / 8$ & $1 / 100$ & $1 / 40$ & \\
\hline$x_{i}$ unconstrained & 1.11 & 2.22 & 3.33 & 4.44 & -0.3510 \\
\hline$x_{2}$ constrained & 0.86 & 2.5 & 2.59 & 3.45 & -0.3622 \\
\hline$x_{1}$ constrained & 2.5 & 2.14 & 3.21 & 4.28 & -0.3576 \\
\hline
\end{tabular}

In the examples above the monotonicity of the optimal unconstrained allocation with respect to the state price density is upset in the optimal VaR constrained allocation. In Fig. 2 the dotted line shows that the optimal VaR constrained portfolio of Example 2 is in fact non-monotonic with respect to the state price density. That is, the investor would be strictly worse off by restricting attention to portfolios that exhibit monotonicity with respect to the state price density. Interestingly, Basak and Shapiro (2001) consider the VaR constrained portfolio problem in a continuous time setting and conclude that the allocation is monotonic with respect to the continuous state price density derived from underlying Brownian motion. Example 2 shows that the discrete case can be qualitatively different.

\section{Conditions for computational simplicity}

Given that the investor's portfolio problem is computationally complex, it is valuable to identify special cases which are easily solvable, i.e., which are of polynomial order.

\subsection{Portfolio insurance}

Portfolio insurance is a special case of Problem 1 where $\delta=0$ which can be solved in polynomial time.

Corollary 1 Portfolio insurance is in the class $P$.

Proof Grossman and Vila (1989) demonstrate that the problem is solved by implementing a put at the desired VaR-level while the consumption in each state is reduced 


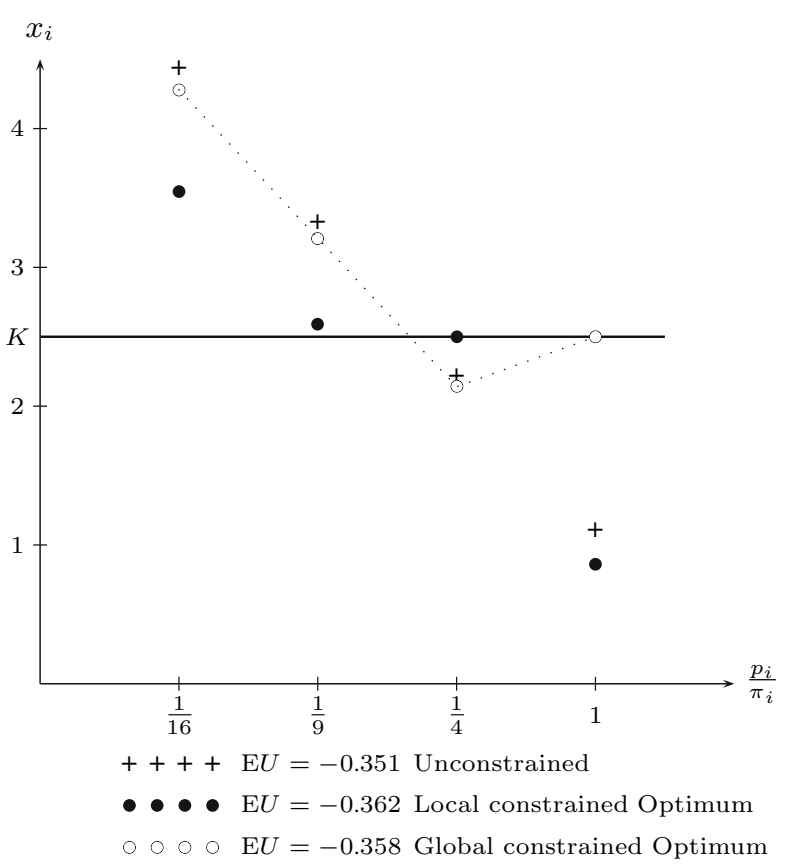

Fig. 2 Monotonicity of solution

to finance this put, in keeping with the first order conditions of the unconstrained problem at the reduced wealth level. Order the state consumption of the unconstrained problem in descending order $x_{1} \geq \cdots \geq x_{n}$. Suppose the VaR restriction becomes binding at $x_{m}<x_{1}$. A sequential search procedure involving at the most $n-m$ steps yields the optimal policy. First check whether the put that ensures consumption at the VaR level in states $m, \ldots, n$ can be financed by reducing the consumption in the remaining uninsured states. If this implies that consumption in state $x_{m-1}$ drops below the VaR level, restart the procedure by insuring consumption in states $m-1, m, \ldots, n$ at the VaR level. Repeat this until the VaR level is met or exceeded in all states.

\subsection{Uniform distribution}

Suppose the probability distribution of the states were discrete uniform, i.e., $\pi_{1}=$ $\pi_{i}, \forall i$. In that case, only the state prices matter, with or without the downside risk constraint in place. Recall Example 1 and Fig. 1, which showed that prices and the VaR level $K$ determine the optimum allocation in the downside risk constrained equilibrium, while prices and probabilities determine the portfolio choice in the unconstrained equilibrium. This latter conclusion is now replaced by recognizing that only prices determine the allocation in both problems. In the unconstrained case if prices are ordered by $p_{1} \leq p_{2} \cdots \leq p_{n}$, then $x_{1} \geq x_{2} \geq \cdots \geq x_{n}$. For the VaR constrained case, the program for finding the optimal solution preserves this monotonicity, and hence 
trivially the monotonicity with respect to the state price density, and is, moreover, easily implemented. To find the optimal VaR constrained allocation, first compute the unconstrained allocation. Subsequently, start tracing the allocation up to $K$ for the state with the lowest price which falls below $K$ in the unconstrained solution. Then turn to the next cheapest state and raise it to $K$. Repeat this procedure until the sum of the probabilities of the (most expensive) states which have $x_{i}<K$ is less than or equal to the desired level $\delta$. This takes at most $n-1$ steps.

Example 3 Continuing Example 1, we expand from two states to three, where state 1 is now divided up into two equally probable states labelled $1 A$ and $1 B$. Each new state costs half the price of the former state 1 security. This new three state economy has state probabilites $\pi_{1 A}=\pi_{1 B}=\pi_{2}=1 / 3$. Assume, as in Example $1, U(x)=\log (x)$, let $W=1 / 4, K=23 / 32, p_{2}=1 / 4$, but $p_{1 A}=p_{1 B}=1 / 6$. It is easily verified that the expected utility, $E U$, of the unconstrained case and the VaR constrained case with $x_{2}=23 / 32$ is identical to the $E U$ in Example 1, since the prices and probabilities of states 1 and 2 in the new example are exactly half the price and probability of state 1 in Example 1.

\begin{tabular}{lllll}
\hline & $x_{1 A}$ & $x_{1 B}$ & $x_{2}$ & $E U$ \\
\hline Unconstrained & $1 / 2$ & $1 / 2$ & $1 / 3$ & -0.83 \\
$x_{2}$ constrained & $27 / 128$ & $27 / 128$ & $23 / 32$ & -1.15 \\
$x_{1 A}$ constrained & $23 / 32$ & $23 / 64$ & $23 / 96$ & -0.93 \\
\hline
\end{tabular}

However, since state 1 is now broken into two cheaper states, while state 2 has the price and probability parameters of Example 1, it is now optimal to place the VaR constraint on state $1 \mathrm{~A}$ (or, alternatively state $1 \mathrm{~B}$ ), rather than raising the consumption of the more expensive state 2 . To meet the VaR constraint, the investor now minimizes the expenditure by raising the allocation in those states which are closest to the VaR level, as these are least costly. Note that this new solution is monotonic with respect to the state price density.

\subsection{Reverse order condition}

We now generalize the previous case of uniform probabilities. Suppose that prices and probabilities are weakly inversely ordered. Note that the previous case of uniform distribution fulfills this inverse order condition. As we argue below, this case may result from financial innovation. Formally the condition is:

Condition 1 Problem 1 satisfies the reverse order condition if there exists a labelling of the states such that the pairs $\left(\pi_{i}, p_{i}\right)$ can be ordered such that

$$
\begin{aligned}
& \pi_{1} \leq \pi_{2} \leq \cdots \leq \pi_{n} \\
& p_{1} \geq p_{2} \geq \cdots \geq p_{n}
\end{aligned}
$$

Remark 1 Note that the reverse ordering condition labelling satisfies the complete market equilibrium condition (3). 
Theorem 2 For any $\delta$, let $q$ be the number such that

$$
\sum_{i=1}^{q} \pi_{i} \leq \delta<\sum_{i=1}^{q+1} \pi_{i}
$$

If $(\pi, p)$ satisfies Condition 1, then Problem 1 can be transformed as follows:

$$
\max \sum_{i=1}^{n} \pi_{i} u\left(x_{i}\right) \text { s.t. } \begin{aligned}
\sum_{i=1}^{n} p_{i} x_{i} & =W \\
0 \leq x_{1} \leq x_{2} \leq \cdots & \leq x_{n} \\
x_{q+1} & \geq K
\end{aligned}
$$

Proof See Appendix A.

Theorem 3 The solution program to (5) is of polynomial order (is in P).

Proof Note that (5) is a linear constrained optimization problem. The feasibility problem of (5) is easy. We need only judge whether the inequality system has solutions. To this end, consider the following linear program,

$$
\begin{aligned}
& \max x_{q+1} \text { s.t } \quad \sum_{i=1}^{n} p_{i} x_{i}=W \\
& 0 \leq x_{1} \leq x_{2} \leq \cdots \leq x_{n}
\end{aligned}
$$

It is straightforward to see that the optimal value of (6) is $W /\left(p_{q+1}+\cdots+p_{n}\right)$. Therefore Problem 1 is feasible under Condition 1 if and only if $W /\left(p_{q+1}+\cdots+p_{n}\right) \geq$ $K$. Consider the following constrained problem:

$$
\begin{aligned}
\max \sum_{i=1}^{n} \pi_{i} u\left(x_{i}\right) \quad \text { s.t } \sum_{i=1}^{n} p_{i} x_{i} & =W \\
x_{i} & \geq 0, \quad i=1,2, \ldots, q \\
x_{i} & \geq K, \quad i=q+1, q+2, \ldots, n
\end{aligned}
$$

If $u(x)$ is strictly concave, following Grossman and Vila (1989), we can prove that there exists $W^{\prime} \leq W$, such that

$$
h_{i}\left(W^{\prime}\right)= \begin{cases}x_{i}\left(W^{\prime}\right) & i=1,2, \ldots, q \\ x_{i}\left(W^{\prime}\right)+\max \left\{K-x_{i}\left(W^{\prime}\right), 0\right\} & i=q+1, q+2, \ldots, n\end{cases}
$$

and $\left\{h_{i}\left(W^{\prime}\right)\right\}$ is an optimal solution of (7). In (8) $\left\{x_{i}\left(W^{\prime}\right)\right\}$ is the optimal solution of the following simple portfolio optimization problem

$$
\max \sum_{i=1}^{n} \pi_{i} u\left(x_{i}\right) \text { s.t } \begin{aligned}
\sum_{i=1}^{n} p_{i} x_{i} & =W^{\prime} \\
x_{1}, x_{2}, \ldots, x_{n} & \geq 0
\end{aligned}
$$


Under Condition 1, we have $0 \leq h_{1}\left(W^{\prime}\right) \leq h_{2}\left(W^{\prime}\right) \leq \cdots \leq h_{n}\left(W^{\prime}\right)$. Thus $\left\{h_{i}\left(W^{\prime}\right)\right\}$ is also a feasible solution of (5). Denote $\Gamma=\left\{x \geq 0 \mid \sum_{i=1}^{n} p_{i} x_{i}=W ; x_{q+1}, \ldots, x_{n} \geq\right.$ $K$ \} the feasible solution set, and denote $\Omega$ the feasible solution set of (5), i.e., $\Omega=$ $\left\{x \geq 0 \mid \sum_{i=1}^{n} p_{i} x_{i}=W ; 0 \leq x_{1} \leq x_{2} \leq \cdots \leq x_{n}, x_{q+1} \geq K\right\}$. It is obvious that $\Omega \subset \Gamma$. We claim that $\left\{h_{i}\left(W^{\prime}\right)\right\}$ is an optimal solution of (5). In fact, since $\Omega \subset \Gamma$, we have $V(\Gamma) \geq V(\Omega)$. Here $V(\Gamma)$ and $V(\Omega)$ are the optimal objective function values. As $\left\{h_{i}\left(W^{\prime}\right)\right\} \in \Omega$, we have $V(\Omega) \geq \sum_{i=1}^{n} \pi_{i} u\left(h_{i}\left(W^{\prime}\right)\right)=V(\Gamma)$. This indicates $\left\{h_{i}\left(W^{\prime}\right)\right\}$ is the optimal solution of (5) as well.

\section{Financial innovation}

In addition to the traditional uses of financial innovation discussed in the introduction, we identify a new application which in general enhances welfare, facilitates the characterization of the optimal portfolio allocation, and reduces complexity. As noted in Sect. 2, the Value-at-Risk constrained utility maximization Problem 1 is computational complex. However, if the reverse order Condition 1 holds, Problem 1 can be solved in polynomial time. This suggests that if we are able to suitably transform the state space to satisfy the reverse order condition, then the transformed Problem 1 will have a polynomial time solution.

\subsection{State splitting}

Consider an economy where the original state space can be augmented by splitting a state into two or more states. State splitting can be achieved by applying a public randomization device when state $i$ arises (such as a roll of dice). This split augments the original state space.

Definition 3 State splitting by an integer factor $\gamma_{i}$ entails dividing state $i$ into $\gamma_{i}$ equally likely sub-states, each of which occur with probability $\pi_{i}^{a}=\pi_{i} / \gamma_{i}$ and cost $p_{i}^{a}=p_{i} / \gamma_{i}$.

Example 4 (Example 1 continued). We now relate Examples 1 and 3. State 1 in Example 1 can be transformed into states $1 A$ and $1 B$ of Example 3 by splitting state 1 in half so that one ends up with $\pi_{1 A}=\pi_{1 B}=\pi_{2}=1 / 3$, while the prices are respectively $p_{1 A}=p_{1 B}=1 / 6, p_{2}=1 / 4$. Thus state splitting for the Example 1 setup, turns this case into Example 3.

Proposition 2 The procedure of state splitting weakly enhances the investor's expected utility

This proposition follows immediately since any portfolio which was feasible prior to state splitting, remains feasible. The increase in welfare may be possible since state splitting relaxes the granularity of the problem so that the VaR constraint becomes less binding. ${ }^{4}$ In the case of Example 3 the expected utility increases with some $20 \%$. Note

\footnotetext{
4 Marin and Rahi (1999) show that randomization may increase welfare due to the Hirshleifer effect that early resolution of uncertainty can limit the availability of insurance.
} 
also that the new solution preserves the monotonicity with respect to the state price density as the reverse ordering conditions holds. Splitting states such that the reverse ordering condition applies is always possible if e.g. the probabilities are rationals. In this case there exists a common denominator $\tau$ for the probabilities of the original state space $\left\{\pi_{i} \mid i=1, \ldots, n\right\}$.

The following is directly implied by the discussion in Sect. 3.2.

Proposition 3 Suppose the state probabilities $\left\{\pi_{i} \mid i=1, \ldots, n\right\}$ have a common denominator $1 / \tau$ for some integer $\tau$. Then each state $i$ can be split by factor $\gamma_{i}=$ $\pi_{i} \tau$, such that the probability distribution function associated with the augmented state space is discrete uniform, $\pi_{i}^{a}=\pi^{a}, \forall i$. The VaR constrained optimal portfolio allocation is then easily characterized since it is monotonic in prices.

We do not however require a common denominator since it is not necessary to render the state space discrete uniform. In general, only some states have to be adjusted to satisfying the reverse ordering condition from Sect. 3.3.

Proposition 4 Suppose there exists a vector of integers $\left(\gamma_{1}, \ldots, \gamma_{n}\right)$ such state splitting implies that the reverse ordering conditions is satisfied. Then the VaR constrained portfolio optimization problem is of polynomial order (is in P).

\section{Proof Apply Theorem 3.}

Since the expected utility of the risk constrained investor's optimal portfolio rises weakly in response to state splitting (as observed by the fact that the Lagrange multiplier associated with the VaR constraint declines), VaR constrained investors are in fact motivated to financial innovation (state splitting) by the risk regulation itself. Hence, the probabilistic type of downward risk constraints provides incentives to financial innovation.

One question unanswered by this theoretical analysis is how state splitting might be implemented in practice. To address this issue, consider an existing class of securities which indeed do split states. Several European government issue bonds which are randomly callable or pay coupons at random. Schilbred (1973) analyzes the case of Italian bonds with random redemption. For the so-called Italian lottery bonds, a predetermined (at the date of issue) percentage of the bond series is retired early each year. An annual lottery determines which particular bonds are retired. This lottery thus introduces exogenous randomness into the economy and effectively creates new states. In a similar vein Green and Rydqvist $(1997,1999)$ analyze random coupon payments for Swedish government bonds, and Ukhov (2002) describes an example of Russian bonds. The prediction suggested by this paper is that the demand for such securities will increase due to the imposition of the VaR constraints by the BIS regulations. We show this by revisiting the examples.

Example 5 We demonstrate the effect of state splitting for the case of the Example 1 portfolio problem. Recall Example 3, and note that since the probabilities are uniform, it splits in effect the Example 1 states in such a way that the reverse ordering condition holds. The unconstrained equilibrium of the Example 1 portfolio is on the $M N-$ segment in Fig. 3, while the suboptimal VaR constrained portfolio is at $N$ and the 


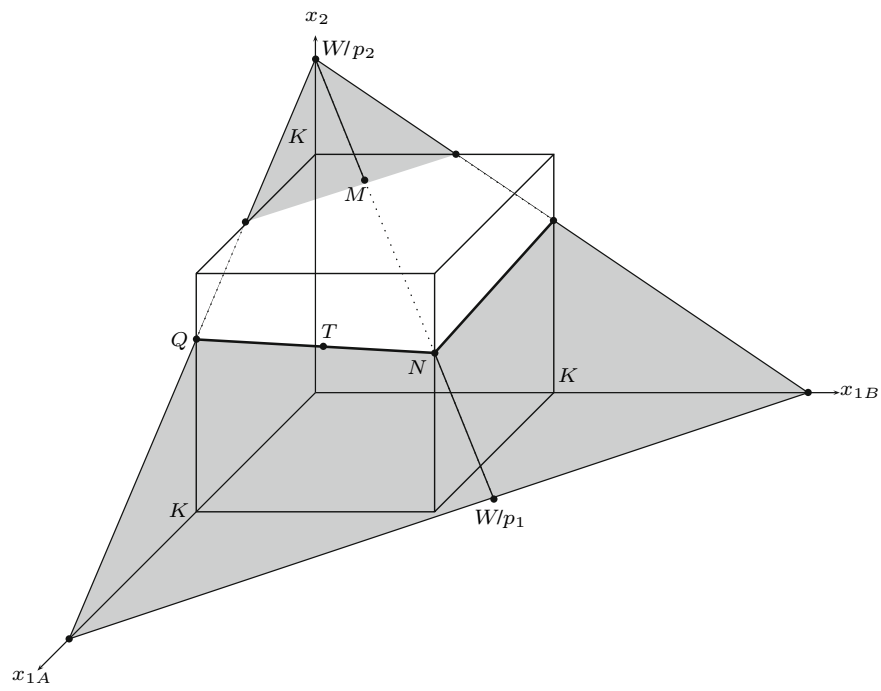

Fig. 3 Impact of state splitting. The cube is the VaR constraint, while the gray plane is the budget constraint

optimal VaR constrained portfolio is at $M$ in terms of Figure 3. Due to state splitting, the calculations in Example 3 indicate it is optimal to move from $M$ in Fig. 3 to the position $T$ exactly half way between $Q$ and $N$ on the line segment $Q N$ of Fig. 3 .

We conclude by illustrating that the reverse ordering condition is not necessary for the optimal VaR constrained portfolio to be monotonic in the state price density.

Example 6 Continuing with Example 2, this problem clearly has too many states to allow depicting these geometrically as we do in Figs. 1 and 3. We have already seen that the optimal VaR constrained solution, in the absence of state splitting, is not monotonic with regards to the state price density. Suppose we split state 2 into two different states $2 A$ and $2 B$ with probabilities $\pi_{2 A}=48 / 100$ and $\pi_{2 B}=2 / 100$ and prices $p_{2 A}=$ $48 / 400$ and $p_{2 B}=2 / 400$ respectively. The optimal VaR constrained portfolio after state splitting entails $\left(x_{1} \approx 1.11, x_{2 A}=2.21, x_{2 B} \approx 2.50, x_{3} \approx 3.32, x_{4} \approx 4.43\right)$ which increases the expected utility to $E U=\sum_{i} \pi_{i} u\left(x_{i}\right) \approx-0.3511$. Indeed, this portfolio is monotonic with respect to the state price density, yet the reverse ordering condition does not hold. However, one could split such that all states are equally probably with $\pi_{i}=1 / 100$ and scale prices accordingly. From this, optimality is established.

\section{Conclusion}

We extend the prior literature on optimal portfolio allocation, subject to a deterministic risk constraint, i.e., portfolio insurance, and allow for the probabilistic VaR constraint. In this case, the optimal portfolio selection problem is computationally complex, and the optimal VaR constrained portfolio solution may not be monotonic with respect to the state price density. 
We further provide a sufficient condition under which the portfolio selection problem becomes computionally simple. Moreover, we demonstrate that investors have an incentive for financial innovation through the introduction of new securities, even though markets are initially complete. The new securities randomize within existing primitive securities and effectively allow investors to better attain the VaR constraint and thus improve expected utility. Furthermore, these securities enable polynomial time solutions.

\section{Proof}

Proof (Proof of Theorem 2) We show that Problem 1 can be transformed into a simple problem if $\pi$ and $p$ satisfy the reverse order relation, that is, $\pi_{i} \leq \pi_{j}$ if and only if $p_{i} \geq p_{j}$. First we claim that there exists an optimal solution $x^{*}$ of Problem 1 such that $0 \leq x_{1}^{*} \leq x_{2}^{*} \leq \cdots \leq x_{n}^{*}$ if $(\pi, p)$ satisfies Condition 1 .

For each feasible solution $x$ of Problem 1 , we denote $(x[1], x[2], \ldots, x[n])$ be a permutation of $(1,2, \ldots, n)$ such that $x_{x[1]} \leq x_{x[2]} \leq \cdots \leq x_{x[n]}$.

Let $x^{*}$ be an optimal solution of Problem 1 . Let $j$ be the smallest subscript such that $\pi_{x^{*}[j]} \geq \pi_{x^{*}[j+1]}$ and $p_{x^{*}[j]} \leq p_{x^{*}[j+1]}$, and at least one of those two inequalities is strict. Let

$$
y=\frac{p_{x *[j]} x_{x *[j]}^{*}+p_{x *[j+1]} x_{x *[j+1]}^{*}}{p_{x *[j]}+p_{x *[j+1]}}
$$

and

$$
z=\frac{p_{x^{*}[j]} x_{x^{*}[j]}^{*}+p_{x^{*}[j+1]} x_{x^{*}[j+1]}^{*}-p_{x^{*}[j]} K}{p_{x^{*}[j+1]}}
$$

Let us construct a new solution $x^{+}$such that

$$
\begin{aligned}
x^{+}[j] & =x^{*}[j+1], x^{+}[j+1]=x^{*}[j] ; \\
x^{+}[i] & =x^{*}[i], \quad i \neq j, j+1 \\
x_{x^{+}[j]}^{+} & =z, x_{x^{+}[j+1]}^{+}=K \quad \text { if } \quad x_{x^{*}[j]}^{*}<K \leq x_{x^{*}[j+1]}^{*} \text { and } y<K \\
x_{x^{+}[j]}^{+} & =y, x_{x^{+}[j+1]}^{+}=y \quad \text { otherwise } \\
x_{x^{+}[i]}^{+} & =x_{x^{*}[i]}^{*}, \quad i \neq j, j+1
\end{aligned}
$$

In the sequel, we show that such a $x^{+}$is an optimal solution of Problem 1 as well. We can easily see that such defined $x^{+}$satisfies the budget constraint.

To prove the optimality of $x^{+}$, we need show that $x^{+}$satisfies the order condition and insurance constraint, and the objective function value of $x^{+}$is not less than the value of $x^{*}$. To this end, we consider three cases separately:

Case $1 x_{x^{*}[j]}^{*}<K \leq x_{x^{*}[j+1]}^{*}$ and $y<K$. 
For this case, we have defined $x_{x^{+}[j]}^{+}=z, x_{x^{+}[j+1]}^{+}=K$. From the definition, we can easily calculate that $z<K \leq x_{x^{*}[j+1]}^{*}$.

Since $p_{x^{*}[j]} \leq p_{x^{*}[j+1]}$ and $x_{x^{*}[j]}^{*}<K$, we have

$$
\left(p_{x^{*}[j]}-p_{x^{*}[j+1]}\right)\left(x_{x^{*}[j]}^{*}-K\right) \geq 0
$$

From (9), we can obtain

$$
x_{x^{*}[j+1]}^{*}+x_{x^{*}[j]}^{*} \leq K+z
$$

Let

$$
\lambda_{1}=\frac{x_{x^{*}[j+1]}^{*}-K}{x_{x^{*}[j+1]}^{*}-x_{x^{*}[j]}^{*}},
$$

and

$$
\lambda_{2}=\frac{x_{x^{*}[j+1]}^{*}-z}{x_{x^{*}[j+1]}^{*}-x_{x^{*}[j]}^{*}}
$$

From (10), we have (i) $x_{x^{*}[j]}^{*} \leq z$, hence the order condition $x_{x^{+}[1]}^{+} \leq x_{x^{+}[2]}^{+} \leq$ $\cdots \leq x_{x^{+}[n]}^{+}$follows from $x_{x^{*}[1]}^{*} \leq x_{x^{*}[2]}^{*} \leq \cdots \leq x_{x^{*}[n]}^{*}$. Further we can easily see that $\sum_{x_{i}^{+} \geq K} \pi_{i} \geq \sum_{x_{i}^{*} \geq K} \pi_{i} \geq 1-\delta$ which follows from $\pi_{x^{+}[j]}=\pi_{x^{*}[j+1]} \leq$ $\pi_{x^{*}[j]}=\pi_{x^{+}[j+1]}$. This indicates that $x^{+}$satisfies the insurance constraint since $x^{*}$ satisfies the insurance constraint.

(ii) $\lambda_{1}+\lambda_{2} \leq 1$. Combine with $u\left(x_{x^{*}[j]}^{*}\right) \leq u\left(x_{x^{*}[j+1]}^{*}\right)$, we have

$$
\left(1-\lambda_{1}\right)\left(u\left(x_{x^{*}[j+1]}^{*}\right)-u\left(x_{x^{*}[j]}^{*}\right)\right) \geq \lambda_{2}\left(u\left(x_{x^{*}[j+1]}^{*}\right)-u\left(x_{x^{*}[j]}^{*}\right)\right)
$$

From the concavity of $u(x)$, we also have

$$
\begin{aligned}
& u(K) \geq \lambda_{1} u\left(x_{x^{*}[j]}^{*}\right)+\left(1-\lambda_{1}\right) u\left(x_{x^{*}[j+1]}^{*}\right) \\
& u(z) \geq \lambda_{2} u\left(x_{x^{*}[j]}^{*}\right)+\left(1-\lambda_{2}\right) u\left(x_{x^{*}[j+1]}^{*}\right)
\end{aligned}
$$

Combine (11) with (12) and (13), we can get

$$
u(K)-u\left(x_{x^{*}[j]}^{*}\right) \geq u\left(x_{x^{*}[j+1]}^{*}\right)-u(z)
$$


Together with $\pi_{x^{*}[j]} \geq \pi_{x^{*}[j+1]}$, we obtain

$$
\begin{aligned}
& \pi_{x^{*}[j]} u\left(x_{x^{*}[j]}^{*}\right)+\pi_{x^{*}[j+1]} u\left(x_{x^{*}[j+1]}^{*}\right) \leq \pi_{x^{*}[j]} u(K)+\pi_{x^{*}[j+1]} u(z) \\
& =\pi_{x^{+}[j]} u\left(x_{x^{+}[j]}^{+}\right)+\pi_{x^{+}[j+1]} u\left(x_{x^{+}[j+1]}^{+}\right)
\end{aligned}
$$

Case $2 x_{x^{*}[j]}^{*} \geq K$ or $x_{x^{*}[j+1]}^{*}<K$.

In this case, we have defined $x_{x^{+}[j]}^{+}=x_{x^{+}[j+1]}^{+}=y$.

First we have

$$
x_{x^{*}[j]}^{*} \leq y \leq x_{x^{*}[j+1]}^{*}
$$

Hence the order condition remains true. Furthermore we have

$$
\sum_{x_{i}^{+} \geq K} \pi_{i}=\sum_{x_{i}^{*} \geq K} \pi_{i} \geq 1-\delta,
$$

the insurance constraint is satisfied.

Next we like to show $x^{+}$remains optimal. For this purpose, we claim that

$$
\sum_{i=1}^{n} \pi_{i} u\left(x_{i}^{+}\right) \geq \sum_{i=1}^{n} \pi_{i} u\left(x_{i}^{*}\right)
$$

Notice that

$$
\begin{aligned}
& \sum_{i=1}^{n} \pi_{i} u\left(x_{i}^{+}\right)-\sum_{i=1}^{n} \pi_{i} u\left(x_{i}^{*}\right) \\
& =\left(\pi_{x^{*}[j]}+\pi_{x^{*}[j+1]}\right) u\left(\frac{\left.p_{x^{*}[j] x_{x^{*}[j]}^{*}+p_{x^{*}[j+1]} x_{x^{*}[j+1]}^{*}}\right)}{p_{x^{*}[j]}+p_{x^{*}[j+1]}}\right) \\
& \quad-\left(\pi_{x^{*}[j]} u\left(x_{x^{*}[j]}^{*}\right)+\pi_{x^{*}[j+1]} u\left(x_{x^{*}[j+1]}^{*}\right)\right),
\end{aligned}
$$

we only need to show that

$$
\begin{aligned}
& \left(\pi_{x^{*}[j]}+\pi_{x^{*}[j+1]}\right) u\left(\frac{p_{x^{*}[j]} x_{x^{*}[j]}^{*}+p_{x^{*}[j+1]} x_{x^{*}[j+1]}^{*}}{p_{x^{*}[j]}+p_{x^{*}[j+1]}}\right) \\
& \geq \pi_{x^{*}[j]} u\left(x_{x^{*}[j]}^{*}\right)+\pi_{x^{*}[j+1]} u\left(x_{x^{*}[j+1]}^{*}\right)
\end{aligned}
$$

Denote $\lambda=\frac{p_{x *[j]}}{p_{x *[j]}+p_{x}{ }^{*}[j+1]}$. Then $0<\lambda<1$. 
From $\pi_{x^{*}[j]} \geq \pi_{x^{*}[j+1]}, p_{x^{*}[j]} \leq p_{x^{*}[j+1]}$, we have $\pi_{x^{*}[j]} p_{x^{*}[j+1]} \geq$ $\pi_{x *[j+1]} p_{x^{*}[j]}$. Furthermore we can deduce that

$$
p_{x^{*}[j]}\left(\pi_{x^{*}[j]}+\pi_{x^{*}[j+1]}\right)-\left(p_{x^{*}[j]}+p_{x^{*}[j+1]}\right) \pi_{x^{*}[j]} \leq 0,
$$

or equivalently

$$
\lambda\left(\pi_{x^{*}[j]}+\pi_{x^{*}[j+1]}\right)-\pi_{x^{*}[j]} \leq 0
$$

From the monotone property of $u(x)$, we have $u\left(x_{x^{*}[j]}^{*}\right)-u\left(x_{x^{*}[j+1]}^{*}\right) \leq 0$. Combine with (16), it follows that

$$
\left[\lambda\left(\pi_{x^{*}[j]}+\pi_{x^{*}[j+1]}\right)-\pi_{x^{*}[j]}\right]\left(u\left(x_{x^{*}[j]}^{*}\right)-u\left(x_{x^{*}[j+1]}^{*}\right)\right) \geq 0
$$

This inequality is equivalent to

$$
\begin{aligned}
& \left(\pi_{x^{*}[j]}+\pi_{x^{*}[j+1]}\right)\left[\lambda u\left(x_{x^{*}[j]}^{*}\right)+(1-\lambda) u\left(x_{x^{*}[j+1]}^{*}\right)\right] \\
& \geq \pi_{x *[j]} u\left(x_{x^{*}[j]}^{*}\right)+\pi_{x^{*}[j+1]} u\left(x_{x^{*}[j+1]}^{*}\right)
\end{aligned}
$$

Notice that $x_{x^{+}[j]}^{+}=x_{x^{+}[j+1]}^{+}=\lambda x_{x^{*}[j]}^{*}+(1-\lambda) x_{x^{*}[j+1]}^{*}$, from the concavity of $u(x)$, we can obtain that

$$
u\left(x_{x^{+}[j]}^{+}\right)=u\left(x_{x^{+}[j+1]}^{+}\right) \geq \lambda u\left(x_{x^{*}[j]}^{*}\right)+(1-\lambda) u\left(x_{x^{*}[j+1]}^{*}\right)
$$

Combine (17) and (18), to get

$$
\left(\pi_{x^{*}[j]}+\pi_{x^{*}[j+1]}\right) u\left(x_{x^{+}[j]}^{+}\right) \geq \pi_{x^{*}[j]} u\left(x_{x^{*}[j]}^{*}\right)+\pi_{x^{*}[j+1]} u\left(x_{x^{*}[j+1]}^{*}\right)
$$

Hence we conclude that $x^{+}$is also an optimal solution of Problem 1.

Case $3 x_{x^{*}[j]}^{*}<K \leq x_{x^{*}[j+1]}^{*}$ but $y \geq K$. In this case, we have also defined $x_{x^{+}[j]}^{+}=$ $x_{x^{+}[j+1]}^{+}=y$. First we have $\sum_{x_{i}^{+} \geq K} \pi_{i}=\sum_{x_{i}^{*} \geq K} \pi_{i}+\pi_{x^{*}[j]} \geq 1-\delta$, thus the insurance constraint is satisfied. Following the same steps as for Case 2, we can show that the order condition remains true, and the objective function value of $x^{+}$is not worse than the objective function value of $x^{*}$. Therefore combining the three cases, we can conclude that there exists an optimal solution $x^{+}$such that $\pi_{x^{+}[j]} \leq \pi_{x+[j+1]}$ and $p_{x+[j]} \geq p_{x+[j+1]}$.

Using the above technique repeatedly, we can always construct an optimal solution of which the order of entries is in the order of $\pi_{i}$, if Problem 1 has an optimal solution. This indicates that there is an optimal solution $x^{*}$ such that

$$
0 \leq x_{1}^{*} \leq x_{2}^{*} \leq \cdots \leq x_{n}^{*}
$$


if $(\pi, p)$ satisfies Condition 1 . Let $q$ be the number determined by (4). We show that $x_{q+1}^{*} \geq K$ for such an optimal solution. In fact, we have $\sum_{x_{i}^{*}<K} \pi_{i} \leq \delta$ by the feasibility of $x^{*}$. If $x_{q+1}^{*}<K$, we get

$$
\sum_{x_{i}^{*}<K} \pi_{i} \geq \sum_{i=1}^{q+1} \pi_{i}>\delta
$$

from (4). This is a contradiction. Conversely if $x$ is a solution such that $\sum_{i=1}^{n} p_{i} x_{i}=$ $W, 0 \leq x_{1} \leq x_{2} \leq \cdots \leq x_{n}$, and $x_{q+1} \geq K$, we have $\sum_{x_{i} \geq K} \pi_{i} \geq \sum_{i=q+1}^{l} \pi_{i} \geq$ $1-\delta . x$ becomes a feasible solution of Problem 1. Hence we can conclude that Problem 1 can be reformulated as $(5)$ when $(\pi, p)$ satisfies Condition 1.

\section{References}

Allen, F., Gale, D.: Optimal security design. Rev Financ Stud 1, 229-263 (1988)

Allen, F., Gale, D.: Arbitrage, short sales and financial innovation. Econometrica 59, 1041-1068 (1991)

Arzac, E.R., Bawa, V.S.: Portfolio choice and equilibrium in capital markets with safety-first investors. J Financ Econ 4, 277-288 (1977)

Basak, S., Shapiro, A.: Value-at-risk based risk management: optimal policies and asset prices. Rev Financ Stud 14, 371-405 (2001)

Chen, Z.: Financial innovation and arbitrage pricing and frictionless economies. J Econ Theory $\mathbf{6 5}, 117-135$ (1995)

Cormen, T.H., Leiserson, C.E., Rivest, R.L.: Introduction to Algorithms. New York/Massachusetts: MIT Press/McGraw Hill (1999)

Dert, C., Oldenkamp, B.: Optimal guaranteed portfolios and the casino effect. Technical Report 9704, Erasmus Center for Financial Research (1997)

Green, R.C., Rydqvist, K.: The valuation of non-systematic risks and the pricing of Swedish lottery bonds. Rev Financ Stud 10(2), 447-480 (1997)

Green, R.C., Rydqvist, K.: Ex-day behavior with dividend preference and limitations to short-term arbitrage: the case of Swedish lottery bonds. J Financ Econ 53(2), 145-187 (1999)

Grinblatt, M., Longstaff, F.: Financial innovation and the role of derivative securities: an empirical analysis of the treasury strips program. J Financ LV(3), 1415-1436 (2000)

Grossman, S., Vila, J.L.: Portfolio insurance in complete markets: a note. J Bus 62(4), 473-476 (1989)

den Hertog, D.: Interior Point Approach to Linear, Quadratic, and Lower Programming. Dortrecht: Kluwer (1995)

Ingersoll, J.E.: Theory of Financial Decision Making. Maryland: Rowman \& Littlefield (1987)

Kuhn, H.W., Tucker, A.W.: Non-linear programming. In: Neyman J (ed) Proceedings of the second berkely symposium on mathematical statistics and probability. Berkeley: University of California Press (1951)

Leland, H.E.: Who should buy portfolio insurance? J Financ 35(2), 581-94 (1980)

Marin, J.M., Rahi, R.: Speculative securities. Econ Theory 14, 653-668 (1999)

Markowitz, H.: Portfolio selection. J Financ 7:77-91 (1952)

Markowitz, H.M.: Portfolio Selection. New York: Wiley (1959)

Miller, M.H.: Financial innovation: the last twenty years and the next. J Financ Quant Anal 21, 459-471 (1986)

Miller, M.H.: Financial innovation: achievements and prospects. J Appl Corp Financ 4, 4-11 (1992)

Papdimitriou, H., Steiglitz, I.: Combinatorial Optimization: Algorithms and Complexity. Englewood Cliffs: Prentice Hall (1982)

Ross, S.A.: Options and efficiency. Q J Econ 90, 75-89 (1976)

Roy, A.D.: Safety first and the holding of assets. Econometrica 20, 431-449 (1952)

Schilbred, C.M.: The market price of risk. Rev Econ Stud 40, 283-292 (1973) 
Ukhov, A.D.: Time varying risk aversion: evidence from russian lottery bonds. Yale School of Management (2002)

Uzawa, H.: The Kuhn-Tucker theorem in concave programming. In: Arrow, K., Hurwicz, L., Uzawa, H. (eds.) Studies in Linear and Non-Linear Programming, Stanford: Stanford University Press (1958)

Vorst, T.: Optimal portfolios under a value at risk constraint. Mimeo, Department of Finance, Erasmus University Rotterdam (2000) 